\title{
A COMPARISION OF VARIOUS EDGE DETECTION TECHNIQUES IN MOTION PICTURE FOR IDENTIFYING A SHARK FISH
}

\author{
${ }^{1}$ Shrivakshan Gopal Thiruvangadan and ${ }^{2}$ Chelliah Chandrasekar \\ ${ }^{1}$ Department of Computer and Applications, \\ Bharathiar University, Coimbatore, Tamilnadu, India \\ ${ }^{2}$ Department of Computer Science, Periyar University, Salem, Tamilnadu, India
}

Received 2013-06-10, Revised 2013-08-05; Accepted 2013-09-18

\begin{abstract}
The significant feature of detecting the motion image objects in this study it try identify the shark fish videos by removing the Background of the image. The main method involved in the detecting from the background is the foreground detection of the image. There are many techniques which usually ignore the fact that the background images consist of different image objects whose conditions may mostly change occur. In this study, a motion picture identification procedure is proposed for real time motion video frames by comparing the three key classes of methods for motion detection primarily the Background Removal (Subtraction) followed by the Temporal distinguishing (differencing) and Optical Flow method. Structured hierarchical background procedure is proposed based on segmenting background images objects. It mainly divided the background images divided into several parts (regions) by the Support Vector Machine (SVM) followed by a structured hierarchical model is built with the region procedure and pixel model procedure. In the region model method, the image object is extracted from the histogram of specific parts which is same to the kind of a Gaussian-combination model. In the pixel model procedure, it is been demonstrated by histograms, picture, which shows gradients sample of pixels in each parts based on the concurrent occurrence of object variations. In this study, it suggests Silhouette detection procedure and it is used. The experimental result are counter validated with a video database to illustrate its efficiencies, which is involved, from static to dynamic scenes by analyzing it with some distinguished motion detection methods chiefly Temporal differencing method followed by Optical Flow method and based on the outputs a motion detection procedure for real time video frames can be created which is cost effective, it shows good rate of accuracy, which is less rate of reliability in simple, less of complexity and well adapted to several kinds of shadow (image) distribution.
\end{abstract}

Keywords: Background Removal (Subtraction), Structure Hierarchical Background Model (HBM), Pixel Model and Region Segmentation

\section{INTRODUCTION}

Background subtraction is a powerful and useful mechanism for detecting many changes in a sequence of objects. There are several approaches for performing the background subtraction procedure. The main method involves the segmentation method (Kumar and Agarwal, 2013). Background images are partitioned into many regions and this partition is done by Mean-shift algorithm. This is from the partition regions it is able to construct a hierarchical model that consists of region model and pixel procedure. The region procedure is mainly extracted from the histogram of a specific region which is similar to the Gaussian mixture procedure. The pixel procedure is based on the image of variation that occurs at the current time series. These variations are Corresponding Author: Shrivakshan Gopal Thiruvangadan, Department of Computer and Applications, Bharathiar University, Coimbatore, Tamilnadu, India 
elaborated by the histogram of each region shown in pixels. As the images change move frequently, the background object locations of background objects are not fixed, so every pixel of the partitioned segmented regions is assigned a weight to denote the probability that this pixel belongs to unique region. The primary difficulty is that they mostly neglect the fact that the background images consist of different objects whose conditions may change more frequently. The more widely used background elimination methods are nonparametric and mixture-of-Gaussian models.

The main task for designing a background exclusion algorithm which is fast is the way of variety done in a detection threshold. The threshold of varying video frames is selected by means of two models. There is usually a nonparametric model defined and in addition to it, a foreground model is introduced. There are two processes (Qin et al., 2012). involved in Background subtraction that work in a loop, background modeling and foreground detection. In background modeling, the model in the view of a camera of the background is created and is periodically updated to handle the changes in illumination. In foreground detection, there involves two decision, first is made as to whether a new intensity fits the background model; second is the resulting label field which is fed back into background modeling to see that no foreground intensities is contaminating the background model. The inclusion of a foreground model tends to grow the detected regions rather than shrinking them. The main disadvantage of this study is that the inclusion may lead to few false positives at the initial label field. According to the functional characteristics of existent time image dispensation systems there were many existing digital image processing algorithms, but the most important Feature to have a sensible hardware and software division for the realization of the functions survive ( $\mathrm{Li}$ et al., 2009). With the basis of software and hardware dissection, a Field Programmable Gate Array (FPGA)-based image processing system structure is built and respectively designs the structure of image acquisition and storage, image processing, real-time display and other functional modules. FPGA consists of four parts that involves Input and Output Module (IOB), routing resources, logic unit, Block Ram.

\subsection{Their Main Functions Are}

- Input and output module: It is defined as the interface of the chip with the outside and is used to complete both the input and output of different electrical characteristics
- Logic unit: It is the main core used to complete all the logic functions

- Routing resource: It is used to connect the logic unit, IOB and Block Ram, mainly to get the good signal broadcast

- Block Ram: It is used to achieve data storage. Finally, the pre-processing circuit that is designed is experimentally verified and the results show that the realization of the hardware design can meet the system functions and their time requiring for processing, which have certain practical value

There is usually need of synchronization between the image acquisition and external trigger events. Hardware and software triggering are widely used although they have several limitations Soft synchronization is investigated in this study to operate by time labeling both the trigger events and images in a video stream and thus by selecting the image matching to each trigger event. For the soft synchronization a stochastic model is developed and, the Fig. (1-7) model proposed in this study was constructed with the inspection application in mind. It therefore incorporates several concepts and features that have proven useful in other object detection algorithms. For instance, many approaches to object detection and shape representation use many tire solution processing to reduce computation while retaining robust results. Which is strand on the model, the uncertainty interval and confidence for correct image selection are determined and an efficient and perfect calibration method is derived. For this method, the images are streamed from the camera to the image processing computer. This is where the images are time tagged and then the image is selected which corresponds to the timing of an external trigger event. This method provides several advantages that include post-triggering capability and natural support for rapidly arriving trigger events and increased flexibility with respect to how the trigger event is sensed and communicated and reduced cost through the opportunity to use a data network interface. The main disadvantage of soft synchronization is the timing decision that is limited by the frame speed. First, the background images which are given as a input are segmented by mean shift segmentation. This technique is based on the mean shift algorithm. For estimating the density gradients, a simple nonparametric procedure is used here. Thus, this program can produce a high quality edge image, or provide, by extracting all the significant colors and a pre-processor for content-based query systems. Gray level images are hand ideas color images having only the lightness coordinate. 
Shrivakshan Gopal Thiruvangadan and Chelliah Chandrasekar / Journal of Computer Science 9 (11): 1427-1434, 2013

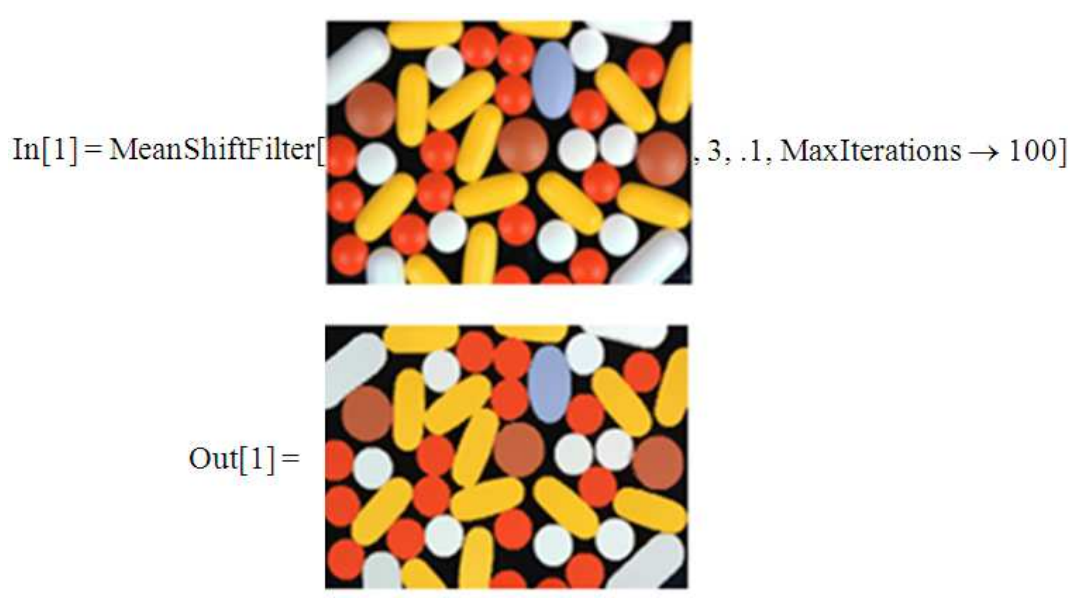

Fig. 1. In this process it replaces every pixel with the mean value of the pixels, pertaining to range-r adjacent neighborhoods, the value is within a range of distance $d$

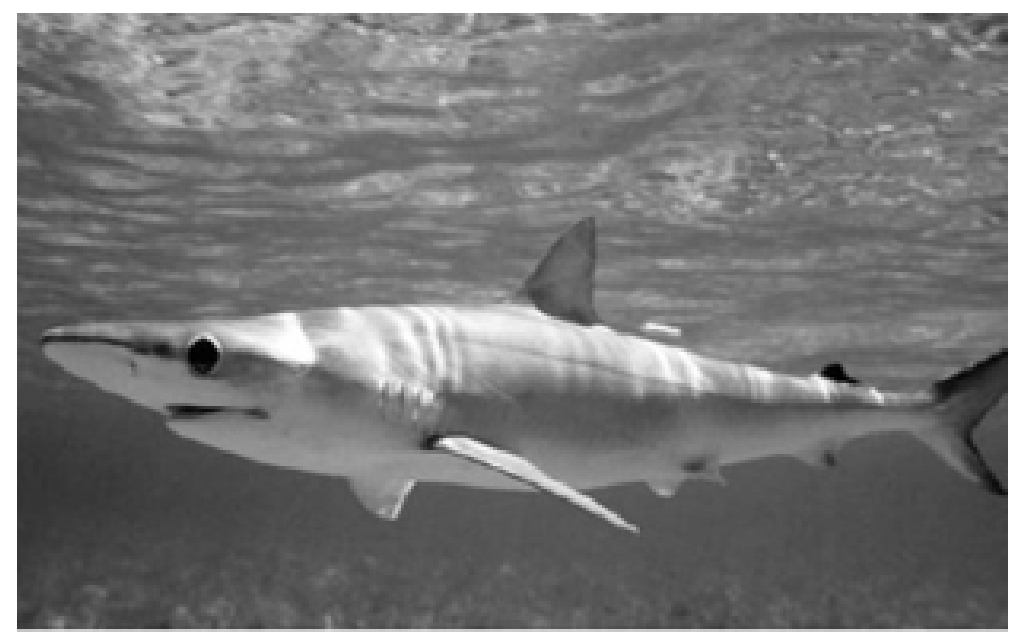

Fig. 2. Shark fish Image with background

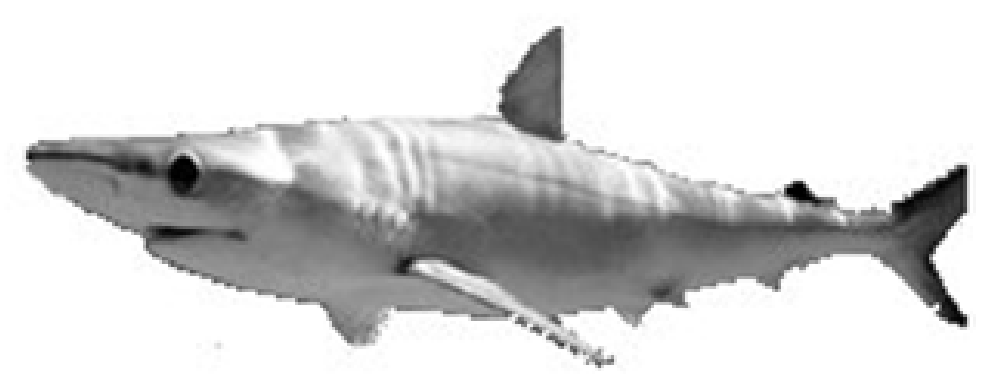

Fig. 3. Shark fish Image subtracted from background 


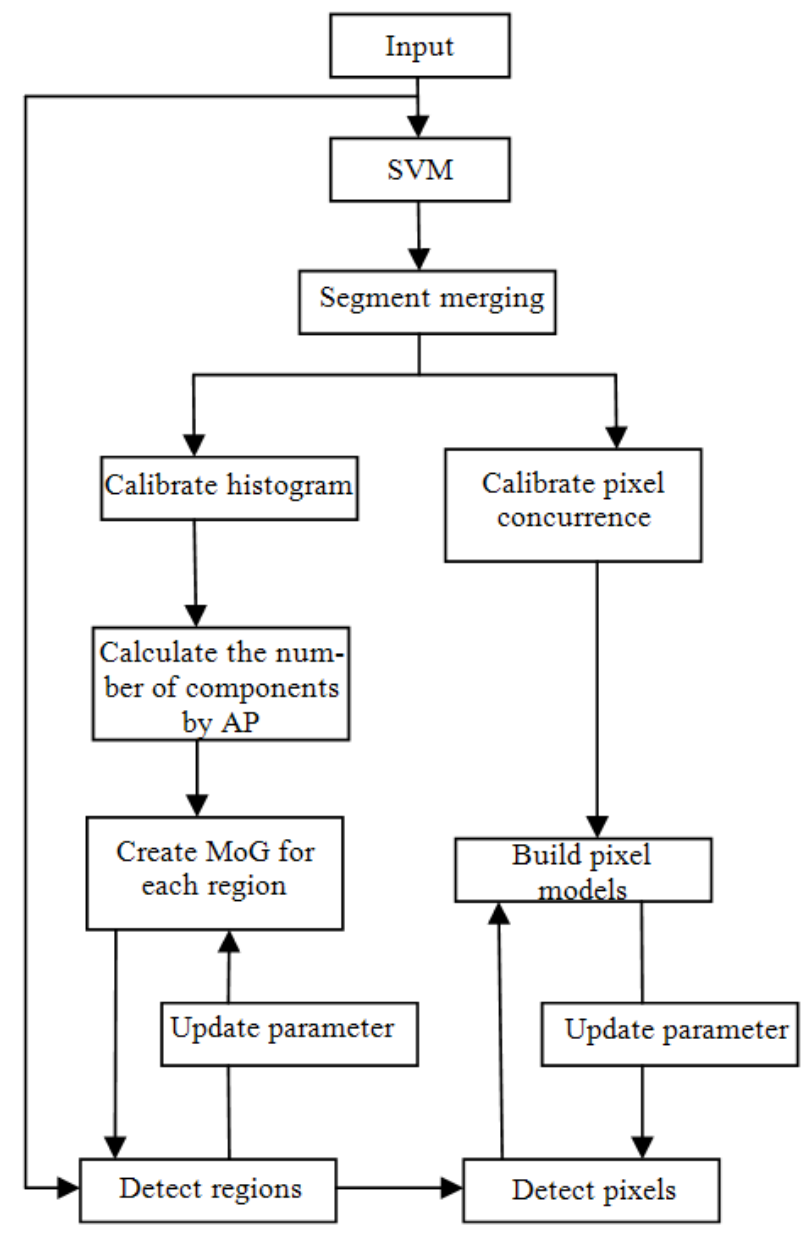

Fig. 4. Architectural design of image detection process

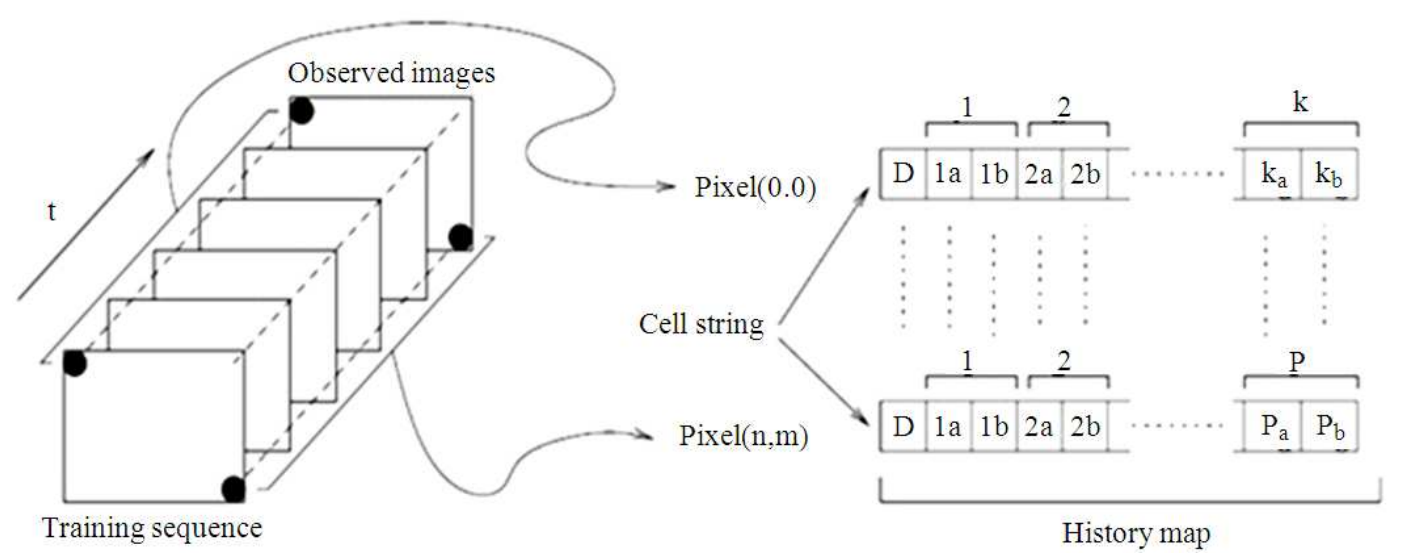

Fig. 5. To calibrate preliminary background model (Left) Training data set sequence, (250-650 frames), (Right) Assessment of the times past map during training data set run for each pixel. $(\mathrm{D}=$ initial cell, $1 \mathrm{a} .1 \mathrm{~b}, \ldots=$ Sub Cells $)$ 


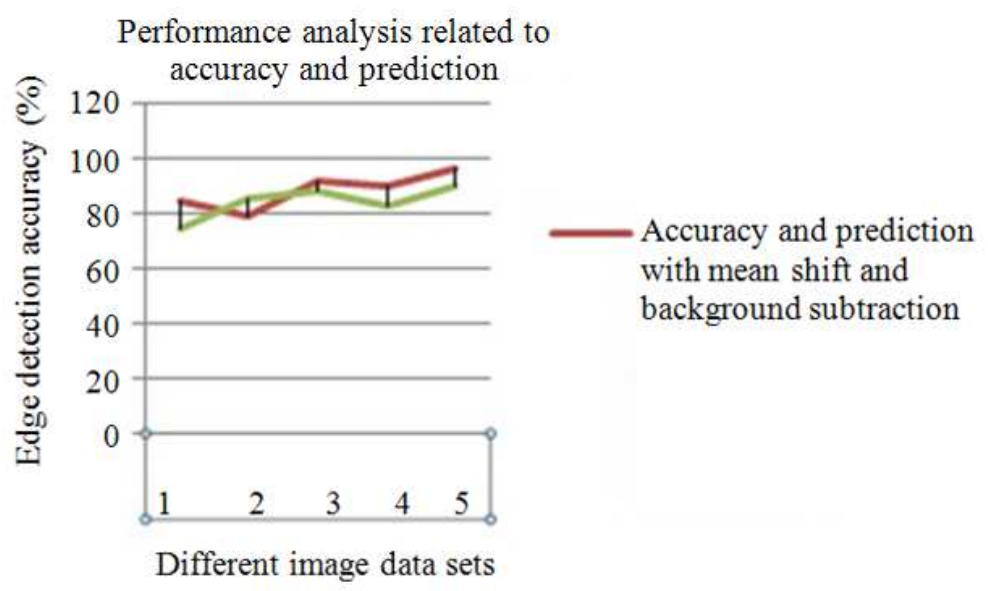

Fig. 6. Performance analysis related to prediction of the shark fish

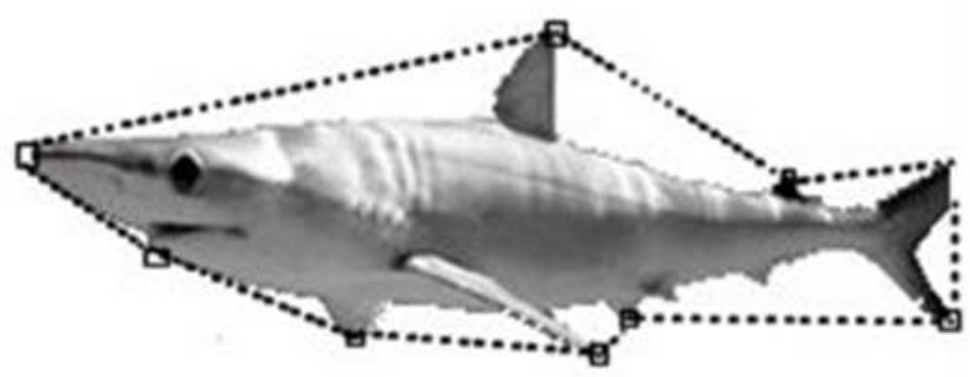

Fig. 7. Edge detection of shark fish after the background removal by mean shift method

In Intelligent moving Systems, the moving shadows were also been wrongly detected as foreground object. This causes bad effect on the latter targets tracking and identify. To minimize the shadows that lie in different regions, there has been implemented a method of moving shadow detection which uses Susan algorithm (Pei and Wang, 2009) based on Image edge detection. This concept of each image point having associated with it a local area of similar brightness is the basis for the SUSAN principle. The local area or SUSAN contains much information about the structure of the image. It is effectively region finding on a small scale. From the size, centroid and second moments of the SUSAN two dimensional features and edges can be detected. This approach to feature detection has many differences to the well known methods, the most obvious being that no image derivatives are used and that no noise reduction is needed. Video highway data is taken with.avi format. Then the edge is detected from Susan method and also from the mixed Gaussian method. Background is obtained by distribution. According to the experiments, SUSAN method is easy to operate and possesses high rate of accuracy, low rate of complexity and well get used to different kinds of shadow distribution.

\subsection{Related Work}

Existing mean Shift is a potent and resourceful non parametric iterative algorithm that can be used for lot of purposes like finding modes, clustering. Mean Shift was introduced in Fukunaga and Hostetler and has been extended to be applicable in other fields like Computer Vision. The main weakness is that they usually neglect the fact that the background images consist of unlike objects whose circumstances may change frequently. The Mean Shift segmentation is a local homogenization method that is very of use for damping shading or tonality differences in local objects. An example is better than many words. 
The Mean Shift takes usually 3 inputs:

- A distance function for measuring distances between pixels. Usually the Euclidean distance, but any other well defined distance function could be used. The Manhattan Distance is another useful choice sometimes

- A radius. All pixels within this radius (measured according the above distance) will be accounted for the calculation

- A worth difference. From all pixels inside radius r, we will take only those whose values are within this difference for calculating the mean

Please note that the algorithm is not well defined at the borders, so dissimilar implementations will give you different results. In this extraction background Subtraction is a process to detect a movement or significant differences inside of the video frame, when compared to a reference and to remove all the nonsignificant components. A Hierarchical copy is developed from the segmented regions of background using Mean-shift algorithm. This Hierarchical model consists of two models, region model and pixel model. The region model is mainly similar to that of the mixture of Gaussian and is chiefly extracted from the histogram of regions which are specific. The pixel model is mainly made up of images that can take place at the same time and are related to each other. The method proposed in this study involves two processing levels. These are the steps taking place in existing system:

- The frames of the video are segmented into regions by mean shift and are taken as the input

- Next, according to their position to form uniform segments for a scene region different frames are merged. When this procedure takes place, a dynamic strategy of representing region borders is also developed, which leads to a more robust performance for dynamic background

- Then the gray value histograms of these regions are computed to build the region models and pixel models are computed by the pixel co occurrence within each region

- The region models are always built as Gaussian mixture models describing the number of components that are determined by a cluster algorithm

- For detecting foreground objects, we first usually segment an input frame according to the uniform segments determined

- Next, each region is detected whether it contains foreground objects by a corresponding region model
If the detected result shows that a region contains foreground objects, first we will detect the pixel belonging to the foreground with the help of pixel models.Secondly after detecting each frame, parameters of region models and pixel models will be updated.

\section{Advantages:}

- It is not necessary that each model must be set constant parameters because assigning unlike, parameters according to the region also leads to a more accurate description

- The weighted pixels in each region make both the descriptor of region and pixels more precise and clear

- The hierarchical models reduces the time cost by just deciding which region contains the foreground and can avoiding other regions that doesn't contain, Because in some dynamic scenes, the locations of background objects are not fixed, each pixel of the segmented regions is assigned a weight to denote the probability that this pixel belongs to one region

\section{Disadvantages:}

- The main disadvantage is Noise. Mean-shift algorithm does not remove the complete noise in the background subtraction

- The second is the shadows. Even the shadows are detected as moving object in the existing system

\subsection{Proposed Solution}

To rise above the problems visage in existing system, we use a advanced technique called Support-Vector Machine for segmenting the background images. The segmented images are meant to form a hierarchical model. This hierarchical model contains region model and pixel model.

The support vector machine is mainly based on statistic learning. They are defined as supervised learning models with associated learning algorithms that can analyze the data and recognize the patterns and also used for classification and regression analysis. It is a new mechanism learning theory. The support vector machine has been widely applied to many applications like pattern gratitude, function approximation and system identification as because support vector machine is able to deal with both the classification and clustering.

\subsection{Classification and Regression by SVM}

Generally the model can be divided into Support Vector Regression and Support Vector Classification. The Training data set is given as $\{(\mathrm{ci}, \mathrm{di})\}^{\mathrm{N}} \quad \mathrm{i}=1$ where ci $\mathrm{Rn}$ and corresponding binary class label biany where ai is the $i^{\text {th }}$ input vector with known binary target bi, Let 
be a non-linear mapping from the data which are original to a high dimensional feature space and it is mainly used to replace sample points ci, cj and they have their mapping images as (ci) and (cj) respectively.

The weight and bias of hyper plane is defined as $\mathrm{w}$ and $b$, respectively. We define the hyper plane which may be ready to act as a decision surface in feature space, as such Equation (1):

$$
\sum_{\mathrm{j}=1}^{\mathrm{i}} \mathrm{w}_{\mathrm{i}} \phi_{\mathrm{j}}+\mathrm{b}=0
$$

First we need to separate the data linearly in the feature space and so the decision function must meet a constraint conditions. The optimization problems are Equation (2):

$$
\begin{aligned}
& \min \operatorname{imize} \phi(\omega, \varepsilon)=\frac{1}{2\|\mathrm{~W}\|^{2}}+c \sum_{\mathrm{i}=1}^{\mathrm{I}} \varepsilon_{\mathrm{i}} \\
& \text { Subject tod }_{\mathrm{i}}\left[\left(\mathrm{W} \cdot \mathrm{c}_{\mathrm{i}}\right)-\mathrm{b}\right] \geq \mathrm{I}-\varepsilon_{\mathrm{i}}
\end{aligned}
$$

where, $\varepsilon_{\mathrm{i}}$ is defined as a stack variable mainly used to relax the margin constraints which are hard. The regular constant $\mathrm{C}>0$ is mainly used to implement the trade-off between the maximal margin of separation and the classification error.

Normally there is a problem of which data cannot be linearly separated in sorting. So to avoid this problem, the Support Vector Machine can map the data which are given as input into a feature space which are high dimensional. Usually the Support Vector Machine constructs an optimal hyper level surface in the high dimensional gap which is transferred into a non- linear decision boundary by first converting it into original space. The non-linear expression for the classification function is given as Equation (3):

$$
t(x)=\sum_{i=1}^{i} a_{i} d_{i}\left(C_{I} \cdot C\right)+b
$$

The routine of SVM also includes the choice of this nonlinear charting function. The SVM applied uses the basis function to perform the operation of mapping. This meaning is expressed in Equation (4):

$$
\mathrm{K}(\mathrm{c}, \mathrm{d})=\exp \left(-\mathrm{s}(\mathrm{c}-\mathrm{d})^{2}\right)
$$

The s parameter in the above equation shows the reflection of the degree of generalization that is made to apply to the data used. Less generalization can be achieved in Support Vector Machine by attaining more data. When there is little s, it may reflect more generalization and a big one reflects less generalization. When the input data is not regulated, this parameter can perform a normalization task. The classification scheme may be also defined with the case of the regression. In this case, the main idea for training the SVM by using d values different from +1 and -1 ..Then, an approximation function is derived that fits approximately the known values only.

\subsection{Silhouette Detection Algorithm}

In this study it also applies Image-space silhouette algorithms. A very simple and useful way to create the silhouettes of a 3D scene is identifying the silhouette edges in the image space. Image space algorithms utilize discontinuities in the image barriers and extract silhouette edges using image giving out methods. The most through way to find silhouettes would be to detect edges in the color buffer, detecting color discontinuous in the image buffer. A big limitation is the image resolution and complicated texture. Highly textured plane will engender many edges that are unrelated to the object shape; likewise, no edge is sensed between two overlapping objects with the equal color. Review all the image space silhouette detecting algorithms, the relatively effective way is detecting silhouettes in special rendered images by developing the geometry information of the 3D scene. The popular knowledge can be categorized into the two classes: The first type algorithms perceive silhouettes in strength map and normal map individually. By compositing the detecting results of depth map and regular map, we can a satisfied last image. Depth map is a system detecting geometry alternating by depth multiplicity.

Silhouette detection Algorithm is mainly used to detect the angle point of the image that is moving. This Algorithm is largely concerned to do the geometry inspection mainly on the basis of image's gray-scale. Then it segregates the pixels into 3 main points. They are angle point, edge point and flat area. To suit a different value when measured in different orders we need to apply about template to figure.

The middle pixel of the pattern is always labeled as nucleus. While sensing the edge, we want to shift the template which is in around to the image and then compare every gray price of the pixel in the pattern along with the nucleus. If the D-value is slighter than the entrance value, mark that this point has like gray-scale to the nucleus Eqution (5):

$\mathrm{q}(\mathrm{w}, \mathrm{w} 0)=\{1|\mathrm{O}(\mathrm{r})-\mathrm{O}(\mathrm{r} 0)|<=\mathrm{d}$

Then $\mathrm{d}=0$, else. 
The parameter $d$ is defined as the threshold value. If the $d$ value is smaller than the threshold value, then that point is marked as to that similar gray-scale to the nucleus. $\mathrm{q}(\mathrm{w}, \mathrm{w} 0)$ is defined as the pixel function. $\mathrm{O}(\mathrm{r} 0)$ is the gray-scale value of the nucleus that is in the center of template. $\mathrm{O}(\mathrm{r})$ is defined as the grayscale value of other pixels in the templates. D is defined as the threshold value.

Therefore, for any image area the pattern goes through, the area which is formed by all the pixels to satisfy the formula (1) called as similar nucleus value area (USAN). The size of USAN area is as follows Equation (6):

$$
\mathrm{n}\left(\mathrm{r}_{0}\right)=\sum \mathrm{q}\left(\mathrm{W}, \mathrm{W}_{0}\right)
$$

There are two main aspects to consider by using Silhouette detection Algorithm for detecting image edge:

- The template selection

- To determine the value for d \& g's threshold value

The two elements used here are used to determine the efficiency of edges that are detected.

\subsection{The Template Selection}

As the image is digitalized, the template found cannot be the real surrounding. Hence to conquer this we use rectangle template $(2 \mathrm{~m}+1) *(2 \mathrm{~m}+1)$ instead.

\subsection{To Determine the Value for $d \& g$ 's Threshold Value}

Threshold value $\mathrm{D}$ is used to determine the contrast ratio of the object and background that are recognizable. Area with slighter contrast ratio, D should be minor

\subsection{Implementation and Experimental Results}

This solution was applied using MATLAB. It is a high-level language and interactive environment for numerical computation, visualization and programming. Using MATLAB, you can analyze data, develop algorithms and create models and function. The language, tools and built in math functions enable you to explore multiple approaches and reach a solution faster than with spreadsheets or traditional programming languages (Sanin et al., 2013). It is important that after the application by the proposed model, has good results over existing techniques. The proposed method's performance is compared with many several available methods. The performance has been observed by calculating noise in the background subtraction.

\section{CONCLUSION}

The objective is to develop a motion detection Technique just to extract foreground objects in a wide range of environmental conditions. Our new technique was designed to handle the problems typically associated with the background subtraction done by hierarchical model. To overcome the problem of noise and shadows an advanced technique called support vector machine for segmenting the background images can be used.The segmented imagery are meant to form a hierarchical model contains region and pixel model. The support vector machine is based on statistic learning. The background subtraction using support vector mechanism can be used to overcome the problem of mistiness and noise and can provide complete feature data.

\section{REFERENCES}

Kumar, K. and S. Agarwal, 2013. An efficient hierarchical approach for background subtraction and shadow removal using adaptive GMM and color discrimination. Int. J. Comput. Applic., 75: 1-7. DOI: $10.5120 / 13160-0752$

Li, J., H. He, H. Man and S. Desai, 2009. A general-purpose fpga-based reconfigurable platform for video and image processing. Adv. Neural Netw., 5553: 299-309. DOI: 10.1007/978-3-642-01513-7_32

Pei, L. and R. Wang, 2009. Moving cast shadow detection based on PCA. Proceedings of the 5th International Conference on Natural Computation, Aug. 14-16, IEEE Xplore Press, Tianjin, pp: 581584. DOI: $10.1109 /$ ICNC.2009.632

Qin, L., J.X. Yu and L. Chang, 2012. Computing structural statistics by keywords in databases. IEEE Trans. Knowl. Data Eng., pp: 1731-1746. DOI: 10.1109/TKDE.2012.78

Sanin, A., C. Sanderson and B.C. Lovell, 2013. Shadow detection: A survey and comparative evaluation of recent methods. Patt. Recogn., 45: 1684-1695. DOI: 10.1016/j.patcog.2011.10.001 